\title{
Lattice-Reduction-Aided Detection with Successive Interference Cancelation for Multiuser Space-Time Block Coded Systems
}

\author{
Tien Duc Nguyen ${ }^{1}$, Quang Duc Le ${ }^{2}$, Xuan Nam Tran ${ }^{3}$, Tadashi Fujino ${ }^{1}$ \\ 1 The University of Electro-Communications, Tokyo, Japan \\ ${ }^{2}$ MoD Institute of Information Technology, 17 Hoang Sam, Cau Giay, Ha Noi, Vietnam \\ ${ }^{3}$ Le Quy Don Technical University, 236 Hoang Quoc Viet, Tu Liem, Ha Noi, Vietnam
}

Correspondence: Xuan Nam Tran, namtx@mta.edu.vn

Manuscript communication: received 20 September 2012, accepted 24 November 2012

\begin{abstract}
Effective detectors with low-complexity are considered for the Alamouti's multiuser space-time block coded (STBC) systems. Viewing the noiseless received signals from $Q$ users as a lattice with basis vectors being the columns of the total channel matrix $H$, we apply lattice reduction to transform the original basis into a nearly orthogonal one which improves the decision regions against noise. Then, linear detection using zero-forcing (ZF) and minimum-mean-square-error (MMSE) methods is performed on the transformed basis to detect transmitted signals from the $Q$ users. These lattice-reduction-aided (LRA) linear detectors significantly improve BER of the linear detectors and, more importantly, allow us to achieve full diversity at high $E b / N_{0}$ regions.
\end{abstract}

Keywords- STBC, multiuser detection, lattice-reduction, zero-forcing, MMSE.

\section{INTRODUCTION}

In multiuser wireless communication systems, besides the effects of channel fading and local noise, the received signal is also interfered by signals from other cochannel users known as co-channel interference (CCI). The objective of detection methods is to detect transmitted signals from individual users in presence of CCI [1]. In terms of minimizing detection error, the maximum likelihood detector (MLD) is optimal, but its excessive complexity prevents it from practical implementation. The linear detectors such as zero-forcing (ZF) or minimum mean square error (MMSE) detectors require less complexity but suffer from BER degradation due to residual interference when the number of users increases [1]. Hence, the goal of multiuser detection problem is to design a reliable detector with reasonable complexity cost. To this direction, recently introduced lattice-reduction-aided (LRA) linear detectors $[2,3]$ are a promising candidate as they can achieve near maximum-likelihood BER at low cost of complexity. Spatial diversity by means of multiple antennas is well-known as an effective way to mitigate channel fading without requiring bandwidth extension. Among spatial diversity techniques, the space-time block code (STBC), introduced by Alamouti [4], is of the most attractive one as it can provide full rate and full diversity with very simple encoding and decoding algorithm. Multiuser detection for the Alamouti's STBC has been recently considered in [5] using MMSE method and improved in [6] using the combined MMSE-ML method. Although the proposed combined MMSE-ML detector of [6] can help to improve the BER of the linear
MMSE detector, its complexity is largely dependent on modulation schemes, thus may not be applied to highorder modulation schemes such as $\mathcal{M}$-QAM or $\mathcal{M}$ PSK for large constellation size $\mathcal{M}$. In order to apply the LRA detection to the multiuser STBC system, in a recent work [7] we proposed two LRA detectors based on the linear ZF and MMSE detection. It was shown in the paper that using the LR helps to improve the BER performance of the detectors significantly. In [8] the authors proposed two LRA detection algorithms based on QR detection, namely, SQRD and AQRD, and presented an implementation of the LRA detectors on programmable device for multiuser STBC systems. The authors of [9], however, proposed a method to reduce the complexity of the LRA detectors for multiuser STBC systems. Unfortunately, this proposal is applicable to a multiuser system with only two users.

In this paper, we generalize our previous work in [7] to the case of multiuser detection with interference successive cancelation (SIC). We will consider both ZF and MMSE in the LRA detection. Our work will serve as a comprehensive case of LRA multiuser detection for STBC systems. The proposed LRA multiuser detectors can work with a system with an arbitrary number of users as long as the number of users does not exceed the number of receive antennas. The proposal of combining SIC with LRA linear detection exhibits significant BER improvement for those systems with large number of users.

The remainder of this paper is organized as follows. In Section 2, system model of a multiuser STBC system is introduced. Section 3 explains fundamentals of LR methods followed by the proposed LRA-ZF, 


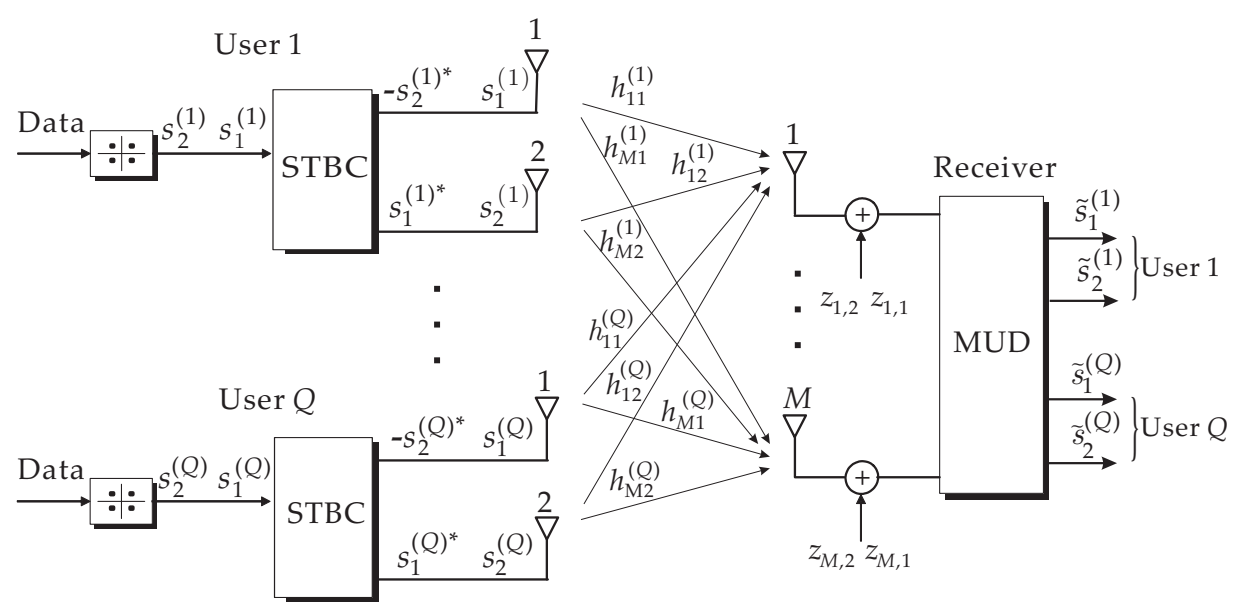

Figure 1. Configuration of Multiuser Detection for STBC transmission

LRA-MMSE and LRA-SIC multiuser detectors for STBC systems. Simulation results are given in Section 6 and conclusion is made in Section 7.

\section{System Model}

We consider a wireless system with $Q$ users using STBC to communicate with the receiver over a flat fading channel as shown in Fig. 1. Although the $Q$ users have been isolated in the figure as in a multiuser system, they can also be considered $Q$ groups of $N_{\mathrm{T}}=2$ transmit antennas in a combined array processing and spacetime coding scheme in [10] or those in a double spacetime transmit diversity (DSTTD) system in [11]. Each user uses the Alamouti's STBC [4] for transmission in order to achieve full-rate and full-diversity. The transmit signal of user $q, q \in\{1,2, \ldots Q\}$, is encoded and transmitted across two antennas and two time slots using the following encoding matrix

$$
S(k)=\left[\begin{array}{ll}
b_{1,1}^{(q)}(k) & b_{2,1}^{(q)}(k) \\
b_{1,2}^{(q)}(k) & b_{2,2}^{(q)}(k)
\end{array}\right]=\left[\begin{array}{cc}
s_{1}^{(q)}(k) & s_{2}^{(q)}(k) \\
-s_{2}^{(q) *}(k) & s_{1}^{(q) *}(k)
\end{array}\right],
$$

where the superscript $(\cdot)^{*}$ denotes the complex conjugation operator, $b_{n, t}^{(q)}(k) \in \mathcal{C}$ is the transmit symbol from antenna $n \in\{1,2\}$ of user $q$ at time slot $t \in\{1,2\}$ during the $k$ th block, and $\mathcal{C}$ is the signal constellation. During the $k$ th block transmission, at the first time slot $t=1$, the first antenna of user $q$ transmits $s_{1}^{(q)}(k)$ while the second antenna transmits $s_{2}^{(q)}(k)$. At the next time slot $t=2$, the first antenna transmits $-s_{2}^{(q) *}(k)$ while the second antenna transmits $s_{1}^{(q) *}(k)$. The receiver is assumed to have an $M_{R}$-element array antenna. Channels between all users and the receivers are quasi-static over a block of some length such that $h_{m n, t}^{(q)}(k)=h_{m n}^{(q)}(k)$. Here $h_{m n, t}^{(q)}(k)$ denotes channel complex gains between transmit antenna $n \in\{1,2\}$ of user $q$ and the receiver antenna $m$ during the $t$ th time slot of the $k$ th transmission block. The received signal at time slot $t$ and the receive antenna $m$ from all $Q$ users is then given by

$$
r_{m, t}(k)=\sum_{q=1}^{Q} \sum_{n=1}^{N_{\mathrm{T}}=2} b_{n, t}(q)(k) h_{m n}^{(q)}(k)+z_{m, t}(k)
$$

with the superscript $(.)^{(q)}$ indicates the expression corresponding to the $q$ th user. Following the method presented in [5] and [6], we build the vector channel model for this multiuser STBC system by defining the following vectors

$$
\begin{aligned}
\underline{\boldsymbol{s}}^{(q)}(k) & \triangleq\left[s_{1}^{(q)}(k), s_{2}^{(q)}(k)\right]^{T}, \\
\underline{\boldsymbol{n}}_{n}^{(q)}(k) & \triangleq\left[h_{1 n}^{(q)}(k), h_{2 n}^{(q)}(k), \ldots, h_{M n}^{(q)}(k)\right]^{T}, \\
\underline{\boldsymbol{r}}_{t}(k) & \triangleq\left[r_{1, t}(k), r_{2, t}(k), \ldots, r_{M, t}(k)\right]^{T}, \\
\underline{\boldsymbol{z}}_{t}(k) & \triangleq\left[z_{1, t}(k), z_{2, t}(k), \ldots, z_{M, t}(k)\right]^{T},
\end{aligned}
$$

where the superscript $(.)^{T}$ denotes the vector transpose operator. Next, stacking $\underline{\boldsymbol{r}}_{t}(k), \underline{\boldsymbol{z}}_{t}(k)$ and $\underline{\boldsymbol{h}}_{n}^{(q)}(k)$, we have

$$
\begin{aligned}
\underline{\boldsymbol{r}}(k) & \triangleq\left[\underline{\boldsymbol{r}}_{1}^{T}(k), \underline{\boldsymbol{r}}_{2}^{H}(k)\right]^{T}, \\
\underline{\boldsymbol{z}}(k) & \triangleq\left[\underline{z}_{1}^{T}(k), \underline{\boldsymbol{z}}_{2}^{H}(k)\right]^{T}, \\
\underline{\boldsymbol{H}}^{(q)}(k) & \triangleq\left[\begin{array}{cc}
\underline{\boldsymbol{h}}_{1}^{(q)}(k) & \underline{\boldsymbol{h}}_{2}^{(q)}(k) \\
\underline{\boldsymbol{h}}_{2}^{(q) *}(k) & -\underline{\boldsymbol{h}}_{1}^{(q) *}(k)
\end{array}\right] .
\end{aligned}
$$

Further, let us construct the input signal vector $\underline{s}(k)$ and the total channel matrix for the multiuser system as $\underline{\boldsymbol{H}}(k)$ as

$$
\begin{aligned}
\underline{\boldsymbol{s}}(k) & \triangleq\left[\underline{\boldsymbol{s}}^{(1) T}(k), \underline{\boldsymbol{s}}^{(2) T}(k), \ldots, \underline{\boldsymbol{s}}^{(Q) T}(k)\right]^{T}, \\
\underline{\boldsymbol{H}}(k) & \triangleq\left[\underline{\boldsymbol{H}}^{(1)}(k), \underline{\boldsymbol{H}}^{(2)}(k), \ldots, \underline{\boldsymbol{H}}^{(Q)}(k)\right],
\end{aligned}
$$

where the the superscript $(.)^{T}$ denotes the Hermitian transpose operator. Ignoring the block index $k$ for notational simplification and using (7)-(11), we have the vector channel model of the considered multiuser STBC system as

$$
\underline{r}=\underline{H s}+\underline{z} .
$$

In order to obtain a lattice representation of the considered system, we shall transform the above complex 
channel vector model into a real-valued model. It is also expected that using the real-valued model would improve BER performance of the non-linear SIC detectors [12]. We begin by constructing the real-valued channel matrix

$$
\boldsymbol{H}=\left[\begin{array}{cc}
\Re\{\underline{\boldsymbol{H}}\} & -\Im\{\underline{\boldsymbol{H}}\} \\
\Im\{\underline{\boldsymbol{H}}\} & \Re\{\underline{\boldsymbol{H}}\}
\end{array}\right]
$$

where the notations $\Re\{$.$\} and \Im\{$.$\} represent the real-$ part and the complex-part operation, respectively. In a similar manner, we convert the transmit signal vector $s$, the receive signal vector $r$, and the noise vector $z$ respectively into the real-valued vectors

$$
\begin{aligned}
& \boldsymbol{s}=\left[\Re\left\{\underline{\boldsymbol{s}}^{T}\right\}, \quad \Im\left\{\underline{\boldsymbol{s}}^{T}\right\}\right]^{T}, \\
& \boldsymbol{r}=\left[\Re\left\{\underline{\boldsymbol{r}}^{T}\right\}, \quad \Im\left\{\underline{\boldsymbol{r}}^{T}\right\}\right]^{T}, \\
& \boldsymbol{z}=\left[\Re\left\{\underline{\boldsymbol{z}}^{T}\right\}, \quad \Im\left\{\underline{\boldsymbol{z}}^{T}\right\}\right]^{T} .
\end{aligned}
$$

The complex channel vector model in (12) now can be converted into a real-valued vector model using equations (13) to (16) as

$$
r=H s+z .
$$

Note that the real-valued matrix $\boldsymbol{H}$ has size $N \times M$ with $N=2 N_{\mathrm{T}} M_{\mathrm{R}}$ and $M=2 Q N_{\mathrm{T}}$, and all the real vectors $x, s, z$ are of an $M \times 1$ column vector.

Equation (17) can be considered a lattice representation where each noiseless received component in $\mathrm{Hs}$ is interpreted as a point of the lattice generated by the generator matrix $\boldsymbol{H}$ while each column of $\boldsymbol{H}$ representing a basis vector. Using this lattice representation we shall apply the idea of lattice basis reduction in [13] to improve decision regions of linear detectors so that their BER performance is enhanced at a cost of very small additional complexity.

In the next section, we shall briefly introduce the idea of lattice basis reduction and its application to the multiuser detection problem of the considered STBC system.

\section{Lattice Basis Reduction}

In order to explain the idea of lattice basis reduction, let us denote columns of the real-valued channel matrix $\boldsymbol{H}$ as $\boldsymbol{h}_{1}, \boldsymbol{h}_{2}, \ldots, \boldsymbol{h}_{N}$. Since channels between all users' antennas and the receiver's antennas are independent, all the vectors $\boldsymbol{h}_{i}, i=1, \ldots, N$, are linearly independent with one another. Therefore, the following combination

$$
\boldsymbol{\Lambda}=c_{1} \boldsymbol{h}_{1}+c_{2} \boldsymbol{h}_{2}+\ldots+c_{N} \boldsymbol{h}_{N} \in \mathbb{R}^{M},
$$

with $N \leq M$ and $c_{i} \in \mathbb{Z}$, can be considered a lattice spanned by the generator matrix $\boldsymbol{H}$. The set of vectors $\left\{\boldsymbol{h}_{1}, \boldsymbol{h}_{2}, \ldots, \boldsymbol{h}_{N}\right\}$ is called a basis of $\boldsymbol{\Lambda}$. Since the transmit vector $s$ contains real elements, we can consider $c_{i}$ as elements of $s$. Then, using the above definition we can view the noiseless component of the receive signal as the lattice, i.e., $\boldsymbol{\Lambda}=\boldsymbol{H}$ s. Note that if $\boldsymbol{H}$ generates $\boldsymbol{\Lambda}$, then any matrix $\hat{H}=H T$, where $\boldsymbol{T}$ is an integer orthogonal matrix with determinant $\operatorname{det}(\boldsymbol{T})= \pm 1$, is another generator matrix of the same lattice $\Lambda$ [14]. $\hat{H}$ can be seen as a transformed generator matrix resulted from a series of operations which do not change the lattice but only its basis. Such operations can be obtained by swapping vectors, subtracting components of one basis vector $\boldsymbol{h}_{j}$ from another $\boldsymbol{h}_{i}$, similar to the GramSchmidt orthogonalization. The orthogonal matrix $T$ is also called a unimodular matrix. It is clear that the inverse of unimodular matrices always exists and contains also only integer values, i.e. $\boldsymbol{T}^{-1} \in \mathbb{Z}^{N}$. Thus, it is followed that the inverse operation $\boldsymbol{H}=\hat{\boldsymbol{H}} \boldsymbol{T}^{-1}$ holds.

The aim of lattice basis reduction, or sometimes simply referred to as lattice reduction (LR), is to transform a given basis $\boldsymbol{H}$ into a new basis $\hat{\boldsymbol{H}}$ with vectors of the shortest length and close to mutually orthogonal. Equivalently, this reduces to find a unimodular matrix $\boldsymbol{T}$ which can make the basis vectors of $\boldsymbol{H}$ nearly orthogonal to one another. There are several lattice basis reduction algorithms available in the literature, among which the algorithm introduced by Lenstra-LenstraLovász [13], abbreviated as the LLL algorithm, is the most popular one because it requires only polynomial complexity. Throughout this paper we shall make use of this algorithm in our lattice-reduction aided (LRA) receivers. The principle of the LLL lattice basis reduction algorithm is briefly introduced below. Readers are referred to [13] for more details on the LLL algorithm. The conventional Gram-Schmidt orthogonalization forms an orthogonal basis $\hat{\boldsymbol{H}}=\left[\hat{\boldsymbol{h}}_{1}, \hat{\boldsymbol{h}}_{2}, \ldots, \hat{\boldsymbol{h}}_{N}\right]$ from the basis $\boldsymbol{H}=\left[\boldsymbol{h}_{1}, \boldsymbol{h}_{2}, \ldots, \boldsymbol{h}_{N}\right]$ by recursively doing for $i$ from 1 to $N$ as

$$
\hat{\boldsymbol{h}}_{i}=\boldsymbol{h}_{i}-\sum_{j=1}^{i-1} \mu_{i j} \hat{\boldsymbol{h}}_{j}
$$

where

$$
\mu_{i j}=\frac{\hat{\boldsymbol{h}}_{j}^{T} \boldsymbol{h}_{i}}{\left\|\hat{\boldsymbol{h}}_{j}\right\|^{2}}
$$

represents the length of projection of $\boldsymbol{h}_{i}$ onto $\hat{\boldsymbol{h}}_{j}$. The operation in the right side of (18) means removing components of $\hat{\boldsymbol{h}}_{1}, \ldots, \hat{\boldsymbol{h}}_{i-1}$ from $\boldsymbol{h}_{i}$, making $\hat{\boldsymbol{h}}_{i}$ orthogonal to other basis vectors.

The basis $\hat{\boldsymbol{H}}=\left[\hat{\boldsymbol{h}}_{1}, \hat{\boldsymbol{h}}_{2}, \ldots, \hat{\boldsymbol{h}}_{N}\right]$ is called LLLreduced if its Gram-Schmidt decomposition satisfies

$$
\left|\mu_{i j}\right| \leq \frac{1}{2} \quad \text { for } \quad 1 \leq j<i \leq N,
$$

and

$$
\delta\left\|\hat{\boldsymbol{h}}_{i-1}\right\|^{2} \leq\left\|\hat{\boldsymbol{h}}_{i}+\mu_{i, i-1} \hat{\boldsymbol{h}}_{i-1}\right\|^{2}
$$

for any $i \in\{2, \ldots, N\}$ and $0<\delta \leq 1$. A common choice of $\delta$ by the LLL algorithm is $\delta=3 / 4$ [3], [13].

One important note from the conventional GramSchmidt orthogonalization is that it changes the lattice, therefore cannot be applied to the lattice reduction. An alternative way is to replace the Gram-Schmidt coefficient $\mu$ by its nearest integer, i.e., $\left\lceil\mu_{i, j}\right\rfloor$, where $\lceil$.$\rfloor denotes rounding operation. This results in weak$ basis reduction which fortunately does not change the lattice. Using a weak basis reduction, the Gram-Schmidt 


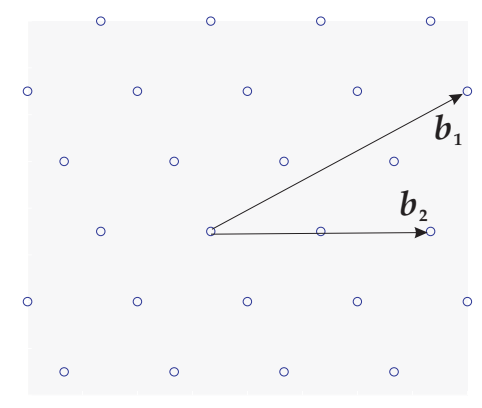

(a) Input basis

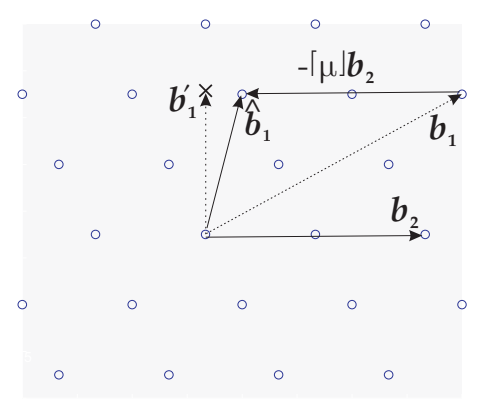

(b) After first iteration

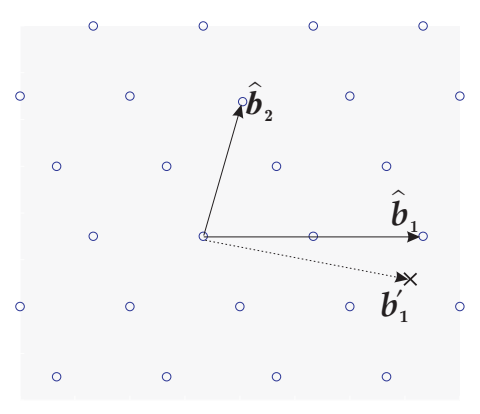

(c) After second iteration

Figure 2. Example of weak reduction in 2D of two vectors $\boldsymbol{h}_{1}$ and $\boldsymbol{h}_{2}$

orthogonalization in (18) becomes

$$
\hat{\boldsymbol{h}}_{i}=\boldsymbol{h}_{i}-\sum_{j=1}^{i-1}\left\lceil\mu_{i j}\right\rfloor \hat{\boldsymbol{h}}_{j},
$$

which satisfies (20). However, only a weak reduction in (22) does not ensure that all the basis vectors have the shortest length as those basis vectors in the front of the sequence $\left\{\hat{h}_{1}, \hat{\boldsymbol{h}}_{2}, \ldots, \hat{\boldsymbol{h}}_{N}\right\}$ may have a longer length than the others. To avoid this situation the LLL lattice basis reduction algorithm checks condition (21) for every $i$. If there is such an $i$ that violates the test condition in (21), the algorithm swaps $\hat{\boldsymbol{h}}_{i}$ and $\hat{\boldsymbol{h}}_{j}$. After swapping the weak reduction is performed again to make sure the two vectors have the shortest length with respect to each other. The algorithm repeats this test and weak reduction process until no more reduction is possible. The modified LLL algorithm which also returns the transform matrix $T$ used in the LRA detectors presented in the next section is shown in Table 3. Note that in the algorithm the notation $\boldsymbol{I}_{M}$ denotes an identity matrix of size $M \times M$.

An illustration of the weak reduction using the LLL algorithm for two two-dimensional (2D) basis vectors is shown in Fig. 2. Note from Fig. 2(b) that the vector $h_{1}^{\prime}$ represents the Gram-Schmidt orthogonalized vector of $\boldsymbol{h}_{1}$ with respect to $\boldsymbol{h}_{2}$. Using the weak reduction, $\mu$ is rounded to the nearest integer and, as a result, $\boldsymbol{h}_{1}^{\prime}$ is shifted to $\hat{\boldsymbol{h}}_{1}$. A second note from the Fig. 2(c) that after the first iteration, the two vector $\hat{h}_{1}$ and $\boldsymbol{h}_{2}$ already formed a weakly reduced basis, thus the second iteration does not help to reduce $\boldsymbol{h}_{2}$ further with respect to $\hat{h}_{1}$. The resulted two vectors $\hat{h}_{1}$ and $\hat{h}_{2}$ become closely orthogonal to each other.

\section{LRA Linear Multiuser Detectors}

In this section, we first briefly introduce two common conventional linear detectors, namely, zero forcing (ZF) and minimum mean square error (MMSE), then apply the application of the lattice reduction to these detectors.
Table I

The Modified LLL-Latticed Basis Reduction Algorithm

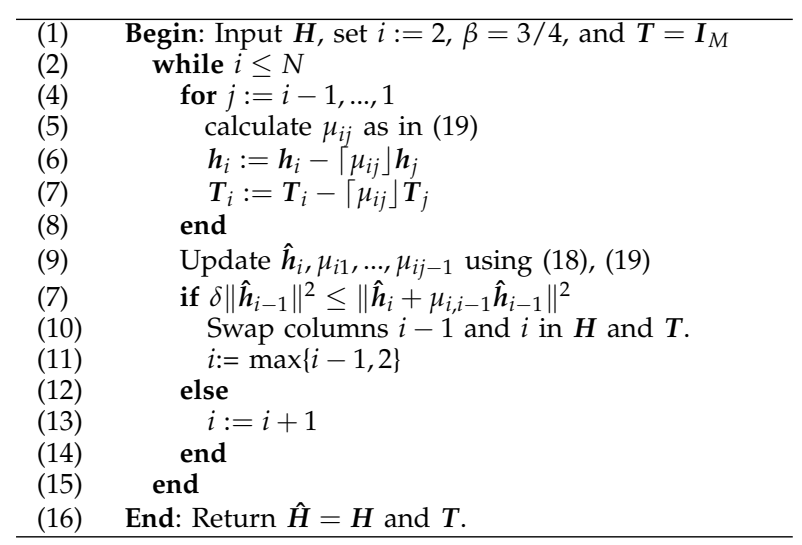

\subsection{Linear Multiuser Detectors}

Using the linear multiuser detector, the estimated vector $\tilde{\boldsymbol{s}}$ of the transmit vector $s$ is given via linear combining the received signal vector $r$ with the weight matrix $W$ as

$$
\tilde{s}=W r
$$

The estimated vector is then quantized to make final decision on the detected signals

$$
\overline{\mathbf{s}}=\mathcal{Q}\{\tilde{\boldsymbol{s}}\}=\mathcal{Q}\{\boldsymbol{W r}\},
$$

where $\mathcal{Q}\{$.$\} denotes the quantization operation.$

4.1.1 ZF Detector: In ZF detectors the linear combining weight matrix is given by the Moore-Penrose pseudo-inverse of the matrix $\boldsymbol{H}$, i.e.

$$
\boldsymbol{W}_{\mathrm{ZF}} \triangleq \boldsymbol{H}^{\dagger}=\left(\boldsymbol{H}^{T} \boldsymbol{H}\right)^{-1} \boldsymbol{H}^{T}
$$

where $(\cdot)^{\dagger}$ represents the pseudo-inverse operation. The estimated vector is thus given by

$$
\tilde{\boldsymbol{s}}_{\mathrm{ZF}}=\boldsymbol{W}_{\mathrm{ZF}} \boldsymbol{r}=\boldsymbol{s}+\left(\boldsymbol{H}^{T} \boldsymbol{H}\right)^{-1} \boldsymbol{H}^{T} \boldsymbol{z} .
$$

Although it is very simple, one problem associated with $\mathrm{ZF}$ detectors is that it suffers from noise amplification which can be seen in the second term of (26). 
4.1.2 MMSE Detector: Different from the ZF detector, the MMSE detector takes into consideration the effect of noise to avoid the problem of noise amplification. The weight matrix of MMSE detector is given by [15]

$$
\boldsymbol{W}_{\mathrm{MMSE}}=\left(\boldsymbol{H}^{T} \boldsymbol{H} \boldsymbol{P}+\sigma^{2} \boldsymbol{I}_{N}\right)^{-1} \boldsymbol{P} \boldsymbol{H}^{T}
$$

where $\sigma^{2}$ is the noise variance and $\boldsymbol{P}=\mathrm{E}\left\{\boldsymbol{s} \boldsymbol{s}^{T}\right\}=$ $\operatorname{diag}\left(\zeta_{1}^{2}, \zeta_{2}^{2}, \ldots \zeta_{N}^{2}\right)$ is the diagonal power matrix. The notation $\mathrm{E}\{$.$\} denotes expectation operator and \operatorname{diag}($. denotes a diagonal matrix with diagonal elements inside the argument. With a simple manipulation, equation (27) can be rewritten as

$$
\boldsymbol{W}_{\mathrm{MMSE}}=\left(\boldsymbol{H}^{T} \boldsymbol{H}+\mathbf{Y}\right)^{-1} \boldsymbol{H}^{T}
$$

where $\mathbf{Y}=\operatorname{diag}\left(\frac{\sigma^{2}}{\zeta_{1}^{2}}, \ldots, \frac{\sigma^{2}}{\zeta_{N}^{2}}\right)$.

In order to simplify our presentation later for both ZF and MMSE detectors, we shall convert the MMSE weight matrix in (27) into a similar form of the ZF in (25) using method presented [16]. For notational simplicity, we shall assume that the average transmit power of all users is the same and equal to $\zeta^{2}$. The MMSE estimated vector $\tilde{\boldsymbol{s}}$ is then given by a new form of weight matrix as

$$
\begin{aligned}
\tilde{\boldsymbol{s}}_{\mathrm{MMSE}} & =\left[\boldsymbol{H}^{T} \boldsymbol{H}+\frac{\sigma^{2}}{\zeta^{2}} \boldsymbol{I}_{N}\right]^{-1} \boldsymbol{H}^{T} \boldsymbol{r} \\
& =\left[\boldsymbol{H}^{T} \boldsymbol{H}+\frac{\sigma^{2}}{\zeta^{2}} \boldsymbol{I}_{N}\right]^{-1}\left[\begin{array}{c}
\boldsymbol{H} \\
\frac{\sigma}{\zeta} \boldsymbol{I}_{N}
\end{array}\right]^{T}\left[\begin{array}{c}
\boldsymbol{r} \\
\mathbf{0}_{N}
\end{array}\right] \\
& =\left(\overline{\boldsymbol{H}}^{T} \overline{\boldsymbol{H}}\right)^{-1} \overline{\boldsymbol{H}}^{T} \overline{\boldsymbol{r}}=\overline{\boldsymbol{H}}^{\dagger} \overline{\boldsymbol{r}}
\end{aligned}
$$

where $\mathbf{0}_{N}$ is a $N$-length zero column vector, $\overline{\boldsymbol{H}}=$ $\left[\boldsymbol{H}, \frac{\sigma}{\zeta} \boldsymbol{I}_{N}\right]^{\top}, \overline{\boldsymbol{r}}=\left[\boldsymbol{r}, \mathbf{0}_{N}\right]^{\top}$, where $(\cdot)^{\top}$ represents the block matrix transposition. The MMSE weight matrix can be now written in a similar form of the $\mathrm{ZF}$ as

$$
\overline{\boldsymbol{W}}_{\text {MMSE }}=\overline{\boldsymbol{H}}^{\dagger}=\left(\overline{\boldsymbol{H}}^{T} \overline{\boldsymbol{H}}\right)^{-1} \overline{\boldsymbol{H}}^{T} .
$$

The original MMSE weight matrix in (27) can be now rewritten as

$$
\boldsymbol{W}_{\mathrm{MMSE}}=\left(\overline{\boldsymbol{H}}^{T} \overline{\boldsymbol{H}}\right)^{-1} \boldsymbol{H}^{T}
$$

\subsection{LRA Linear Detectors}

The principle of LRA linear detectors is first to find a lattice reduced basis $\hat{H}$ of the original basis (channel matrix) $\boldsymbol{H}$ and then apply the linear detection methods on the obtained reduced basis. Let us rewrite the system model vector in (17) as

$$
r=H s+z=H T T^{-1} s+z=\hat{H} u+z
$$

where $u=T^{-1} s$. Since $T$ contains integer elements, for $s \in \mathbb{Z}^{N}$ we also have $\boldsymbol{u} \in \mathbb{Z}^{N}$. Moreover, as both $\hat{\boldsymbol{H}} \boldsymbol{u}$ and $H s$ describe the same point in the lattice, the object of the LRA linear detection is to detect $u$ instead of $s$. If we denote $\tilde{u}$ the estimates of $u$, then we obtain the estimates of $s$ using the transform matrix $T$ as $\tilde{\boldsymbol{s}}=\mathbf{T} \tilde{\boldsymbol{u}}$.

The proposed LRA-ZF and LRA-MMSE detectors are presented below.
4.2.1 LRA-ZF Detector: Similar to (25), the ZF weight matrix used to detect $u$ is given by

$$
W_{\mathrm{LRA}-\mathrm{ZF}}=\hat{H}^{\dagger} \text {. }
$$

The decision statics (estimates) of $\boldsymbol{u}$ are given by

$$
\tilde{u}_{\mathrm{LRA}-\mathrm{ZF}}=\boldsymbol{W}_{\mathrm{LRA}-\mathrm{ZF}} \boldsymbol{r}=\hat{\boldsymbol{H}}^{\dagger} \boldsymbol{r}=\boldsymbol{u}+\hat{\boldsymbol{H}}^{\dagger} \boldsymbol{z} .
$$

Since $\boldsymbol{u} \in \mathbb{Z}^{N}$, it is intuitive to perform an unconstrained elementwise quantization, $\bar{u}_{\mathrm{LRA}-\mathrm{ZF}}=$ $\mathcal{Q}\left\{\tilde{\boldsymbol{u}}_{\mathrm{LRA}-\mathrm{ZF}}\right\}$, then calculate $\tilde{\boldsymbol{s}}_{\mathrm{LRA}-\mathrm{ZF}}=\boldsymbol{T} \tilde{\boldsymbol{u}}_{\mathrm{LRA}-\mathrm{ZF}}$, and finally restrict this result to the signal constellation. Note that before applying the quantization we need to properly shift and scale $\tilde{\boldsymbol{u}}_{\text {LRA-ZF }}$ such that it really contains integer value. Depending on the specific constellation, the shifting and scaling step can be different. With 4-QAM, for example, if the transmitted signal is from the constellation $\mathcal{A}:\left\{ \pm \frac{1}{\sqrt{2}} \pm \frac{1}{\sqrt{2}} j\right\}$ then the vector $s$ will contain elements with value of $\left\{ \pm \frac{1}{\sqrt{2}}\right\}$. And $\mathbf{s}$ presents for the integer vector $\hat{s}$, whose each element $\hat{s} \in\{0,1\}$ is shifted by $\frac{1}{2}$ and scaled by $\sqrt{2}$ as below

$$
\boldsymbol{s}=\sqrt{2}\left(\hat{\boldsymbol{s}}-\left[\frac{1}{2}, \cdots, \frac{1}{2}\right]^{T}\right) .
$$

Thus,

$$
\boldsymbol{u}=\boldsymbol{T}^{-1} \boldsymbol{s}=\sqrt{2} \boldsymbol{T}^{-1} \hat{\boldsymbol{s}}-\boldsymbol{T}^{-1}\left[\frac{1}{\sqrt{2}}, \cdots, \frac{1}{\sqrt{2}}\right]^{T} .
$$

Now, the quantization step consists of shifting and scaling $u$ in order to allow simple element-rounding for vector $\left(\boldsymbol{T}^{-1} \hat{\mathbf{s}}\right)$ whose elements are expected to be integer, and then, shifting and scaling the rounded vector back to get the estimate $\tilde{u}$ of $u$ as

$$
\begin{aligned}
\tilde{u}=\sqrt{2} \mathcal{Q}\left\{\frac { 1 } { \sqrt { 2 } } \left(\boldsymbol{u}+\boldsymbol{T}^{-1}\right.\right. & {\left.\left.\left[\frac{1}{\sqrt{2}}, \cdots, \frac{1}{\sqrt{2}}\right]^{T}\right)\right\} } \\
& -T^{-1}\left[\frac{1}{\sqrt{2}}, \cdots, \frac{1}{\sqrt{2}}\right]^{T} .
\end{aligned}
$$

For LRA-ZF detector, the quantization step is given as

$$
\begin{gathered}
\tilde{u}_{\mathrm{LRA}-\mathrm{ZF}}=\sqrt{2} \mathcal{Q}\left\{\frac{1}{\sqrt{2}}\left(\boldsymbol{u}_{\mathrm{LRA}-\mathrm{ZF}}+\boldsymbol{T}^{-1}\left[\frac{1}{\sqrt{2}}, \cdots, \frac{1}{\sqrt{2}}\right]^{T}\right)\right\} \\
-\boldsymbol{T}^{-1}\left[\frac{1}{\sqrt{2}}, \cdots, \frac{1}{\sqrt{2}}\right]^{T} .
\end{gathered}
$$

When we get the estimate $\tilde{u}$ the transmitted signal is estimate by $\tilde{s}_{\mathrm{LRA}-\mathrm{ZF}}=T \tilde{\boldsymbol{u}}_{\mathrm{LRA}-\mathrm{ZF}}$, Due to the error detection the estimated elements of $\tilde{s}_{\mathrm{LRA}-\mathrm{ZF}}$, which are not in the set points of $\left\{ \pm \frac{1}{2}\right\}$, have to be snapped to one point by comparing to zero decision boundary.

4.2.2 LRA-MMSE Detector: Although a suboptimal MMSE receiver can reduce the noise enhancement by allowing some residual spatial interference, the overall improvement is incremental. A straight forward way to improve performance of the MMSE detector is to replace $\boldsymbol{H}$ by $\hat{\boldsymbol{H}}$ in (28) to get the improved weight matrix. However, doing that will not give the weight matrix the full benefit from lattice reduction since the LR application to $\boldsymbol{H}$ does not take the noise term into account. However, if we assume the LLL-reduced 
$\hat{\boldsymbol{H}}=\overline{\boldsymbol{H}} \overline{\boldsymbol{T}}$, which means $\overline{\boldsymbol{H}}=\overline{\boldsymbol{T}}^{-1} \hat{\boldsymbol{H}}$, from the MMSE weight matrix in (30) we have

$$
\begin{aligned}
\tilde{\boldsymbol{s}}_{\mathrm{MMSE}} & =\left(\overline{\boldsymbol{H}}^{T} \overline{\boldsymbol{H}}\right)^{-1} \overline{\boldsymbol{H}}^{T} \overline{\boldsymbol{r}} \\
& =\left[\left(\overline{\boldsymbol{T}}^{-1} \hat{\boldsymbol{H}}\right)^{T}\left(\overline{\boldsymbol{T}}^{-1} \hat{\boldsymbol{H}}\right)\right]^{-1}\left(\overline{\boldsymbol{T}}^{-1} \hat{\boldsymbol{H}}\right)^{T} \overline{\boldsymbol{r}} \\
& =\overline{\boldsymbol{T}}\left((\hat{\boldsymbol{H}})^{T} \hat{\boldsymbol{H}}\right)^{-1}(\hat{\boldsymbol{H}})^{T} \overline{\boldsymbol{r}}=\overline{\boldsymbol{T}}(\hat{\boldsymbol{H}})^{+} \overline{\boldsymbol{r}} .
\end{aligned}
$$

From this expression, we get $\boldsymbol{u}_{\mathrm{LRA}-\mathrm{MMSE}}=\overline{\boldsymbol{T}}^{-1} \tilde{\boldsymbol{s}}_{\mathrm{MMSE}}$, which is similar to the LRA-ZF detection but for the MMSE. Since $\hat{\boldsymbol{H}}$ is LLL-reduced matrix of $\overline{\boldsymbol{H}}$, multiplying the noisy receive vector with $\hat{\mathbf{H}}^{\dagger}$ causes much less noise amplification compared to that of $\hat{\boldsymbol{H}}^{\dagger}$. Moreover, since the estimate is calculated according to the MMSE detection, $u_{\text {LRA-MMSE }}$ will exploit the benefit from both LR and noise amplification avoidance to improve the MMSE detection performance.

Note that the expression of the MMSE detector in (29) is similar to that of the ZF detector and, thus, that the orthogonality of $\overline{\mathbf{H}}$, not that of $\mathbf{H}$, will decide the noise amplification level. Therefore, we should apply the method for LRA-ZF to the pseudo-channel matrix $\overline{\mathbf{H}}$ to have the LRA-MMSE detector and to allow LLLreduced matrix $\hat{\mathbf{H}}$ to fully exploit both advantages from the MMSE detection and lattice reduction, therefore, achieve improved performance. This means that we will first perform the LR for the matrix $\bar{H}$, i.e. $\hat{\boldsymbol{H}}=\overline{\boldsymbol{H}} \bar{T}$, then compute $\boldsymbol{u}_{\text {LRA-MMSE }}=\hat{\boldsymbol{H}}^{\dagger} \overline{\boldsymbol{r}}$. The quantization step is similar to that for LRA-ZF detector as

$$
\begin{aligned}
\tilde{\boldsymbol{u}}_{\mathrm{LRA}-\mathrm{MMSE}} & \\
=\sqrt{2} & \mathcal{Q}\left\{\frac{1}{\sqrt{2}}\left(\boldsymbol{u}_{\mathrm{LRA}-\mathrm{MMSE}}+\overline{\boldsymbol{T}}^{-1}\left[\frac{1}{\sqrt{2}}, \cdots, \frac{1}{\sqrt{2}}\right]^{T}\right)\right\} \\
& -\overline{\boldsymbol{T}}^{-1}\left[\frac{1}{\sqrt{2}}, \cdots, \frac{1}{\sqrt{2}}\right]^{T} .
\end{aligned}
$$

\section{Combined LRA Linear Detectors AND Successive InTERference Cancellation}

This section will focus on the principle of the standard nonlinear multiuser detection technique, namely Successive Interference Cancellation (SIC), as well as the more recent advances in reduced complexity version SIC by using QR decomposition. We then develop LRASIC detection for the multiuser STBC systems.

\subsection{Principle of SIC}

The SIC detection algorithm is based on the solution of linear ZF- or MMSE detection, but the principle is to detect one user at a time and not in parallel. The user with the highest post detection signal-to-noiseratio (SNR) or smallest error covariance is detected first. Once this user has been detected, his signal is subtracted from the received signals of all users, following which the user with the next highest SNR is detected. This process is repeated until all the users have been detected.

Recall from previous sections, using (26) and (31) the covariance matrix for the estimation error of ZF- and
MMSE detection is given by

$$
\mathrm{E}\left\{\left(\boldsymbol{s}-\tilde{\boldsymbol{s}}_{\mathrm{ZF}}\right)\left(\boldsymbol{s}-\tilde{\boldsymbol{s}}_{\mathrm{ZF}}\right)^{T}\right\}=\sigma^{2}\left(\boldsymbol{H}^{T} \boldsymbol{H}\right)^{-1}
$$

and

$$
\begin{aligned}
\mathrm{E}\left\{\left(\boldsymbol{s}-\tilde{\boldsymbol{s}}_{\mathrm{MMSE}}\right)\left(\boldsymbol{s}-\tilde{\boldsymbol{s}}_{\mathrm{MMSE}}\right)^{T}\right\} & =\sigma^{2}\left[\boldsymbol{H}^{T} \boldsymbol{H}+\frac{\sigma^{2}}{\zeta^{2}} \boldsymbol{I}_{n}\right]^{-1} \\
\text { or } & =\sigma^{2}\left(\overline{\boldsymbol{H}}^{T} \overline{\boldsymbol{H}}\right)^{-1} \cdot(42)
\end{aligned}
$$

It is clear that the estimation error of each element of $s$ will be proportional to the diagonal element of the matrix. Therefore, the first detected signal among the entries of $s$ will be the one with the smallest diagonal element of the covariance matrix, i.e., the one with the smallest error covariance. It is not difficult to see that if the first detected element is the $i$ th entry of $s$ then the norm of the columns $\boldsymbol{h}_{i}$ of $\boldsymbol{H}$ will be maximum compared to the other columns. After estimation of $\tilde{s}_{i}$, considering $\tilde{s}_{i}$ as a known quantity, we obtain the canceled signal vector $\boldsymbol{r}-\boldsymbol{h}_{i} \tilde{s}_{i}$ and reduced order channel matrix $\boldsymbol{H}^{(n-1)}$, which is the remaining $n-1$ columns. Then, the whole procedure has to be repeated again to detect each element one by one. Due to the effect of error propagation it is very important to detect in optimal order direction. Thus two main steps of SIC detection are:

1) Determine the optimal detection order and the weight vector/matrix.

2) Obtain the linear estimate of the signal with the highest post-detection SNR, quantize the estimate and cancel the effect of the sliced estimate signal.

Since step 1 contains the major complexity of SIC detection with inverting and squaring the matrix $\boldsymbol{H}$ or $\overline{\boldsymbol{H}}$, as well as the requirement of pseudo-inversion of the deflated channel matrix in each stage, it is necessary to investigate the advanced method to achieve optimal detection order and weight matrix with reduced complexity.

\subsection{ZF-SIC with Optimal Ordering}

In order to avoid inverting and squaring matrix operation in the order sorting step, the usage of $Q R$ decomposition was introduced in [16] and [17] with significant complexity reduction. In this section, we reconsider the ZF-SIC and the post optimal sort algorithm in detail and try to improve it. Let us start with the QR decomposition of the extended channel matrix

$$
H=Q R
$$

where $Q$ is an $n \times n$ orthonormal matrix and $\boldsymbol{R}$ is an $n \times n$ upper triangular matrix. From this decomposition the error covariance matrix in (41) can be rewritten as

$$
\begin{aligned}
\boldsymbol{\Phi}_{\mathrm{ZF}} & =\sigma^{2}\left(\boldsymbol{H}^{T} \boldsymbol{H}\right)^{-1}=\sigma^{2}\left[(\boldsymbol{Q R})^{T}(\boldsymbol{Q R})\right]^{-1} \\
& =\sigma^{2} \boldsymbol{R}^{-1} \boldsymbol{R}^{-T}
\end{aligned}
$$

and the ZF solution in (26) can be rewritten as

$$
\begin{aligned}
\tilde{\boldsymbol{s}}_{\mathrm{ZF}} & =\left[(\boldsymbol{Q R})^{T}(\boldsymbol{Q R})\right]^{-1}(\boldsymbol{Q R})^{T} \boldsymbol{r}=\boldsymbol{R}^{-1} \boldsymbol{R}^{-T} \boldsymbol{R}^{T} \boldsymbol{Q}^{T} \boldsymbol{r} \\
& =\boldsymbol{R}^{-1} \boldsymbol{Q}^{T} \boldsymbol{r} .
\end{aligned}
$$


Because $\boldsymbol{R}^{-1}$ has an upper triangular form (46) is given by

$$
\left[\begin{array}{c}
\tilde{s}_{1}^{\mathrm{ZF}} \\
\cdot \\
\cdot \\
\tilde{s}_{n}^{\mathrm{ZF}}
\end{array}\right]=\left[\begin{array}{ccccc}
R_{1,1}^{-1} & \cdot & \cdot & \cdot & \cdot \\
0 & \cdot & \cdot & \cdot & \cdot \\
0 & 0 & R_{i, i}^{-1} & \cdot & \cdot \\
0 & 0 & 0 & \cdot & \cdot \\
0 & 0 & 0 & 0 & R_{n, n}^{-1}
\end{array}\right]\left[\begin{array}{c}
\boldsymbol{q}_{1}^{T} \boldsymbol{r} \\
\boldsymbol{q}_{i}^{T} \boldsymbol{r} \\
\cdot \\
\boldsymbol{q}_{n}^{T} \boldsymbol{r}
\end{array}\right]
$$

where $R_{i, i}^{-1}$ is the $(i, i)$ th element of $\boldsymbol{R}^{-1}$ and $R_{i, i}$ is the $(i, i)$ th element of $\boldsymbol{R} ; \boldsymbol{q}_{i}$ is the $i$ th column of $\boldsymbol{Q}$. Thus, from two equation (44) and (47) we can have the following two important observations:

- The estimate error is proportional to the row norm of $R^{-1}$ and thus, to minimize estimate error at each SIC-stage, the corresponding entry associated with the minimum norm row of $\boldsymbol{R}^{-1}$, or maximum norm column of $\boldsymbol{R}$, should be detected first.

- To avoid pseudoinverse operation at every SICstage the ZF solution can be detected from the last layer to the first layer as

$$
\tilde{s}_{n}^{\mathrm{ZF}}=\frac{1}{\bar{R}_{n, n}} \boldsymbol{q}_{n}^{T} \boldsymbol{r}
$$

and canceled from received signal vector as

$$
\boldsymbol{r}=\boldsymbol{r}-\boldsymbol{h}_{n} \tilde{s}_{n}^{\mathrm{ZF}} .
$$

Therefore, by permuting entries of $s$ or equivalent exchanging column of $\boldsymbol{H}$ should guarantee an optimum ordered matrix $\boldsymbol{H}_{\text {ordered }}$ such that the $\boldsymbol{R}_{\text {ordered }}^{-1}$ with the minimum row norm will be the last row of any submatrix $K \times K$ of $\boldsymbol{R}_{\text {ordered }}^{-1} K=n, \ldots, 2$. That is, in the optimal sorting algorithm, instead of inverting and squaring $H$, it reduces to find the minimum norm row of $\boldsymbol{R}^{-1}$ and its submatrix and reorder the entry to the last row together with exchanging corresponding column of $\boldsymbol{H}$. Note that exchanging columns of $\boldsymbol{H}$ is done together with exchanging corresponding rows in $\boldsymbol{R}^{-1}$, keeping the relation $\boldsymbol{H}=Q R$ still holds but exchanging operation will output a matrix $\boldsymbol{R}_{e x}^{-1}$ without the triangular structure. To recover the triangular structure one can use a unitary Householder matrix $\Theta$ as suggested in [17]. It comes from the our observation that

$$
\begin{aligned}
\boldsymbol{H}_{e x} & =\boldsymbol{Q}_{e x} \boldsymbol{R}_{e x}=\boldsymbol{Q}_{e x} \boldsymbol{\Theta} \boldsymbol{\Theta}^{-1} \boldsymbol{R}_{e x} \\
& =\left(\boldsymbol{Q}_{e x} \boldsymbol{\Theta}\right)\left(\boldsymbol{R}_{e x}^{-1} \boldsymbol{\Theta}\right)^{-1}=Q_{\text {new }}\left(\boldsymbol{R}_{\text {new }}^{-1}\right)^{-1} .
\end{aligned}
$$

Due to the multiplication with $\Theta$ the matrix $\boldsymbol{R}_{\text {new }}^{-1}$ has the last row with only one element similar as in (47) and can be used for the next step. Once the last row is properly sorted, the procedure is repeated for the near last row $n-1$ of the $(n-1) \times(n-1)$ submatrix of $\boldsymbol{R}_{\text {new }}^{-1}$ and so on to get the $\boldsymbol{H}_{\text {ordered }}$. Improvement can be made by observing that the relation $\boldsymbol{H}_{\text {ordered }}=$ $Q_{\text {ordered }} \boldsymbol{R}_{\text {ordered }}$ holds, thus, it is unnecessary to update $Q$ for every exchanging step as in [17]. We can obtain $Q_{\text {ordered }}$ in the end by

$$
\boldsymbol{Q}_{\text {ordered }}=\boldsymbol{H}_{\text {ordered }} \boldsymbol{R}_{\text {ordered }}^{-1}
$$

where, $\boldsymbol{H}_{\text {ordered }}$ and $\boldsymbol{R}_{\text {ordered }}^{-1}$ are certainly known.
We can now summarize the ZF-SIC algorithm as follows

1) Compute $Q$ and $R^{-1}$ by QR decomposition of $\boldsymbol{H}$.

2) Find the minimum norm row of $\boldsymbol{R}^{-1}$ and permute it to be the last $n$th row. Permute $s$ and columns of $\boldsymbol{H}$ in $\boldsymbol{H}$.

3) Calculate the Householder matrix $\Theta$ to make $\boldsymbol{R}_{e x}^{-1} \boldsymbol{\Theta}$ become block upper triangular.

4) Go back to step 2 but now calculate with $(n-1) \times$ $(n-1)$ submatrix of $\boldsymbol{R}^{-1}$. Repeat step 2, 3, 4 until submatrix reduces to $1 \times 1$.

5) From here, we get $\boldsymbol{H}_{\text {order }}$, calculate $\boldsymbol{Q}$ as in (50).

6) Apply ZF-detection for each entry and cancel the signal effect with order from $n$th to 1 st as in (48) and (49).

More details on how to calculate $\Theta$ can be found in [17].

\subsection{LRA-ZF-SIC Detector}

Similar to the linear detection, we can consider the lattice-reduced version of the system model with the equivalent channel matrix $\hat{H}=\hat{Q} \hat{R}$ and the transmitted signal $u=T^{-1} s$, leading to LRA-ZF-SIC detection with the decision variables given by

$$
\tilde{\boldsymbol{s}}_{\text {LRA-ZF-SIC }}=\hat{\boldsymbol{R}}^{-1} \hat{\mathbf{Q}}_{1}^{T} \boldsymbol{r} .
$$

Note that the quantization step is applied for each detected element. The properly scaling and shifting is described in [18]. The LRA-ZF-SIC algorithm is then rewritten from ZF-SIC algorithm as

1) Apply lattice-reduction to get $\hat{\boldsymbol{H}}=\boldsymbol{H T}$.

2) Apply the optimal sorting algorithm for $\hat{\boldsymbol{H}}$ as described from step 1 to 4 of ZF-SIC algorithm.

3) From here, we obtain $\hat{\boldsymbol{H}}_{\text {order }}$, calculate $\hat{\boldsymbol{Q}}$ as in (50).

4) Apply LRA-ZF-detection and quantization for each entry as in (51) and then cancel the signal effect with order from $n$th to 1st as in (49), but in terms of $z$ instead of $s$.

5) Calculate the original $\tilde{\boldsymbol{s}}=\mathcal{Q}\{\boldsymbol{T} \tilde{\boldsymbol{z}}\}$. The transmitted signal from each user can be then reconstructed by doing inversion of (15) and (10).

\subsection{MMSE-SIC with Optimal Ordering}

It is interesting to note that there is a difference between the sorting algorithm above from the algorithm in [16] and [17] in that the one we introduce can be used for either ZF-SIC or MMSE-SIC with only replacing $\overline{\boldsymbol{H}}$ for $\boldsymbol{H}$ and vice versa, thanks to the ZF-detection similar form of MMSE-detection in (29). Indeed, similar to ZF-SIC let us start with the QR decomposition of the extended channel matrix

$$
\overline{\boldsymbol{H}}=\left[\begin{array}{c}
\boldsymbol{H} \\
\sigma \mathbf{I}_{n}
\end{array}\right]=\overline{\mathbf{Q}} \overline{\boldsymbol{R}}=\left[\begin{array}{l}
\boldsymbol{Q}_{1} \\
\boldsymbol{Q}_{2}
\end{array}\right] \overline{\boldsymbol{R}}
$$

where $\bar{Q}$ is an $(n+m) \times n$ orthonormal matrix and $\bar{R}$ is an $n \times n$ upper triangular matrix. From this decomposition the error covariance matrix in (42) can be rewritten as

$$
\begin{aligned}
\boldsymbol{\Phi}_{\mathrm{MMSE}} & =\sigma^{2}\left[\overline{\boldsymbol{H}}^{T} \overline{\boldsymbol{H}}\right]^{-1}=\sigma^{2}\left[(\overline{\mathbf{Q}} \overline{\mathbf{R}})^{T}(\overline{\mathbf{Q}} \overline{\mathbf{R}})\right]^{-1} \\
& =\sigma^{2} \overline{\mathbf{R}}^{-1} \overline{\mathbf{R}}^{-T}
\end{aligned}
$$


and the MMSE solution in (31) can be rewritten as

$$
\begin{aligned}
\tilde{\boldsymbol{s}}_{\text {MMSE }} & =\left[(\overline{\mathbf{Q}} \overline{\boldsymbol{R}})^{T}(\overline{\mathbf{Q}} \overline{\boldsymbol{R}})\right]^{-1}\left(\boldsymbol{Q}_{1} \overline{\boldsymbol{R}}\right)^{T} \boldsymbol{r} \\
& =\overline{\boldsymbol{R}}^{-1} \overline{\boldsymbol{R}}^{-T} \overline{\boldsymbol{R}}^{T} \boldsymbol{Q}_{1}^{T} \boldsymbol{r} \\
& =\overline{\boldsymbol{R}}^{-1} \boldsymbol{Q}_{1}^{T} \boldsymbol{r} .
\end{aligned}
$$

Because $\overline{\boldsymbol{R}}^{-1}$ has an upper triangular form (55) is given by

$$
\left[\begin{array}{c}
\tilde{s}_{1}^{\mathrm{MMSE}} \\
\cdot \\
\cdot \\
\dot{\tilde{s}_{n}^{\mathrm{MMSE}}}
\end{array}\right]=\left[\begin{array}{ccccc}
\bar{R}_{1,1}^{-1} & \cdot & \cdot & \cdot & \cdot \\
0 & \cdot & \cdot & \cdot & \cdot \\
0 & 0 & \bar{R}_{i, i}^{-1} & \cdot & \cdot \\
0 & 0 & 0 & \cdot & \cdot \\
0 & 0 & 0 & 0 & \bar{R}_{n, n}^{-1}
\end{array}\right]\left[\begin{array}{c}
\boldsymbol{q}_{1}^{T} \boldsymbol{r} \\
\boldsymbol{q}_{i}^{T} \boldsymbol{r} \\
\dot{\boldsymbol{q}_{n}^{T}} \boldsymbol{r}
\end{array}\right]
$$

where $\bar{R}_{i, i}^{-1}$ is the $(i, i)$ th element of $\overline{\boldsymbol{R}}^{-1}$ and equal $\frac{1}{\bar{R}_{i, i}}$; $\boldsymbol{q}_{i}$ is the $i$ th column of $Q_{1}$.

Thus, from two equation (53) and (56) we can have similar observations for ZF-SIC. Like ZF-SIC, the corresponding entry associated with the minimum norm row of $\overline{\boldsymbol{R}}^{-1}$ should be detected first and the MMSE solution should be detected in the direction from the last layer to the first layer as

$$
\tilde{s}_{n}^{\mathrm{MMSE}}=\frac{1}{\bar{R}_{n, n}} \boldsymbol{q}_{n}^{T} \boldsymbol{r}
$$

and canceled from received signal vector as

$$
\boldsymbol{r}=\boldsymbol{r}-\overline{\boldsymbol{h}}_{n} \tilde{\mathrm{s}}_{n}^{\mathrm{MMSE}} .
$$

Therefore, by permuting entries of $s$ or equivalent exchanging column of $\boldsymbol{H}$ should guarantee an optimum ordered matrix $\overline{\boldsymbol{H}}_{\text {ordered }}$ such that the $\overline{\boldsymbol{R}}_{\text {ordered }}^{-1}$ with the minimum row norm will be the last row of any submatrix $K \times K$ of $\overline{\boldsymbol{R}}_{\text {ordered }}^{-1} K=n, \ldots, 2$. That is, in the optimal sorting algorithm, instead of inverting and squaring $\bar{H}$, it reduces to find the minimum norm row of $\overline{\boldsymbol{R}}^{-1}$ and its sub matrix and then reorders the entry to the last row together with exchanging corresponding column of $\boldsymbol{H}$.

Note that as mentioned before, due to the fact that the relation $\overline{\boldsymbol{H}}_{\text {ordered }}=\bar{Q}_{\text {ordered }} \overline{\boldsymbol{R}}_{\text {ordered }}$ holds for every steps, it is unnecessary to update $Q_{1}$ for every exchanging step as in [17]. Finally, we can obtain $Q_{1}$ as

$$
\boldsymbol{Q}_{1}=\boldsymbol{H}_{\text {ordered }} \overline{\boldsymbol{R}}_{\text {ordered }}^{-1}
$$

where, $\boldsymbol{H}_{\text {ordered }}$ and $\overline{\boldsymbol{R}}_{\text {ordered }}^{-1}$ are certainly known.

We can now summarize the MMSE-SIC algorithm as follows

1) Compute $\bar{Q}$ and $\overline{\boldsymbol{R}}^{-1}$ by QR decomposition of $\overline{\boldsymbol{H}}$.

2) Find the minimum norm row of $\overline{\boldsymbol{R}}^{-1}$ and permute it to be the last $n$th row. Permute $s$ and columns of $\boldsymbol{H}$ in $\overline{\boldsymbol{H}}$ also.

3) Calculate the Householder matrix $\Theta$ to make $\overline{\boldsymbol{R}}_{e x}^{-1} \boldsymbol{\Theta}$ become block upper triangular.

4) Go back to step 2 but now calculate with $(n-1) \times$ $(n-1)$ submatrix of $\overline{\boldsymbol{R}}^{-1}$. Repeat step 2, 3, 4 until the submatrix reduces to $1 \times 1$.

5) From here, we get $\overline{\boldsymbol{H}}_{\text {order }}$, calculate $Q_{1}$ as in (59).

6) Apply MMSE-detection for each entry and cancel the signal effect with order from $n$th to 1 st as in (57) and (58).

\subsection{LRA-MMSE-SIC}

Similar to the linear detection, we can consider the lattice-reduced version of the extended system model with the equivalent channel matrix $\hat{\boldsymbol{H}}=\hat{\hat{Q}} \hat{\mathbf{R}}$ and transmitted signal $u=T^{-1} s$, leading to LRA-MMSESIC detection with the decision variables given by

$$
\tilde{\mathbf{s}}_{\text {LRA-MMSE-SIC }}=\hat{\overline{\mathbf{R}}}^{-1} \hat{\mathbf{Q}}_{1}^{T} \boldsymbol{r} \text {. }
$$

Note that the quantization step is applied for each detected element. The properly scaling and shifting is described in [18]. Similar to the LRA-ZF-SIC, the LRAMMSE-SIC algorithm is then rewritten from MMSE-SIC algorithm as

1) Apply lattice-reduction to get $\hat{\boldsymbol{H}}=\overline{\boldsymbol{H}} T$.

2) Apply the optimal sorting algorithm for $\hat{\boldsymbol{H}}$.

3) From here, we obtain $\hat{\boldsymbol{H}}_{\text {order }}$, calculate $\hat{Q}_{1}$ as in (59).

4) Apply LRA-MMSE-detection and quantization for each entry as in (60) and then cancels the signal effect with order from $n$th to 1st as in (58), but in term of $z$ instead of $s$.

5) Calculate the original $\tilde{s}=\mathcal{Q}\{T \tilde{z}\}$. The transmitted signal from each user can be then reconstructed by doing inversion of (14) and (10).

\section{Simulation and Results}

We simulate a multiuser Alamouti's STBC system with $K=4$ users and a 4 -antenna receiver. The transmit data are 4-QAM modulated with the average transmit power per bit equal to $E_{b}$. The elements of channel matrix $\boldsymbol{H}$ and noise $\mathbf{z}$ is Gaussian distributed with variance equal to one and $\sigma^{2}$, respectively. At each antenna of the receiver, the SNR per bit of each user is $\mathrm{SNR}=E_{b} / \sigma^{2}=2 \zeta^{2} / \sigma^{2}$. Using this relation, we can calculate the noise variance and simulate the noise for different SNR at each $R x$ antenna.

Figures 3 and 4 illustrate the BER performance of the linear ZF and MMSE detector for the case with and without using LR with different multiuser scenarios. It is clear that for all scenarios the use of the LR helps to improve the BER performance dignificantly for both the $\mathrm{ZF}$ and MMSE detection. Comparing the slopes of the BER curves we can also see that with the assistance of LR, the linear detectors can achieve the same diversity order of the single detector.

Figure 5 compares BER performance of the ZF and MMSE linear detectors. We can realize from the figure is that the MMSE detectors outperform the ZF for all cases of simulation. This is clear by using the fact that the MMSE detection can avoid the problem of noise amplification of the ZF detection. However, the difference is smaller for case with smaller number of users. For example, for the case of 4 users, the difference is about $2 \mathrm{~dB}$ but this reduces to less than $1 \mathrm{~dB}$ for the case of 3 or 2 users. This can be explained using the LR characteristics. It is easy to understand that for the case with less users, after LR the channel matrix will be easier to be reduced, i.e. the channel matrix will 


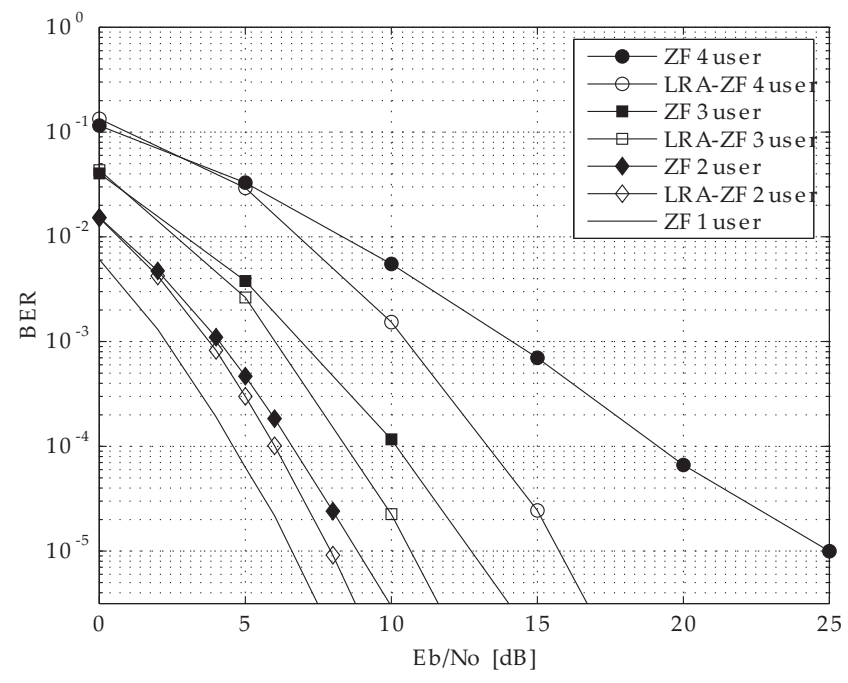

Figure 3. BER performance of linear ZF detection.

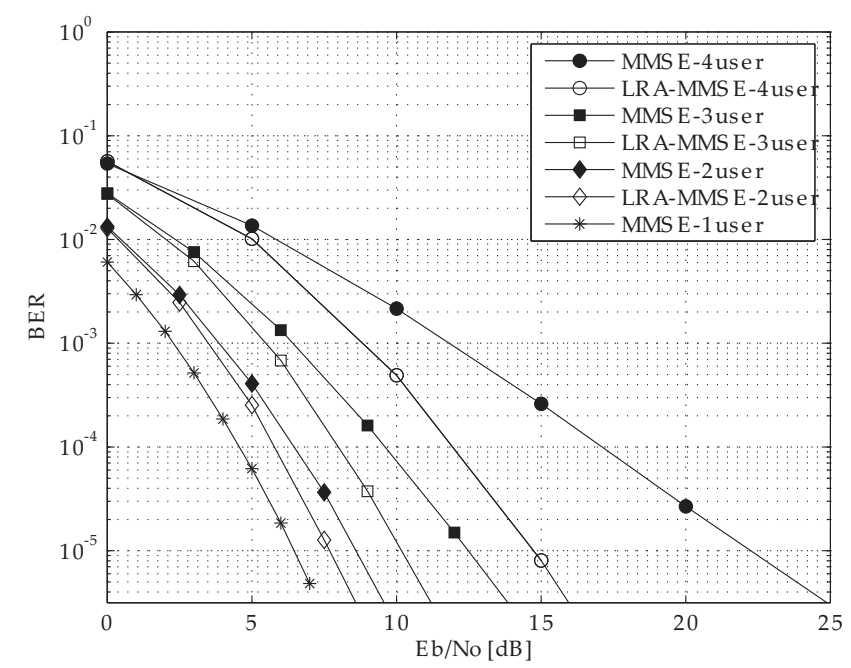

Figure 4. BER performance of linear MMSE detection.

become closer to orthogonal. As a result the detection boundary will be improved and thus we can have better BER performance.

Finally, we illustrate the efficacy of the SIC detectors by showing the BER performance of the MMSE detector in Fig. 6. It can be seen from the figure that we can have significant BER performance improvement thanks to use of SIC with the LRA-MMSE detection, however, only for the case with more users. For the case with less users, the LRA-MMSE is almost optimal and thus the use of SIC does not achieve further improvement.

\section{Conclusions}

In this paper, we have applied the lattice reduction method to the multiuser detection of the Alamouti's STBC systems and proposed two LRA linear detectors based on ZF and MMSE detection with successive interference cancelation. We have showed that with the help of lattice reduction, both the LRA-linear detectors can achieve more diversity, and thus provide better BER performance.

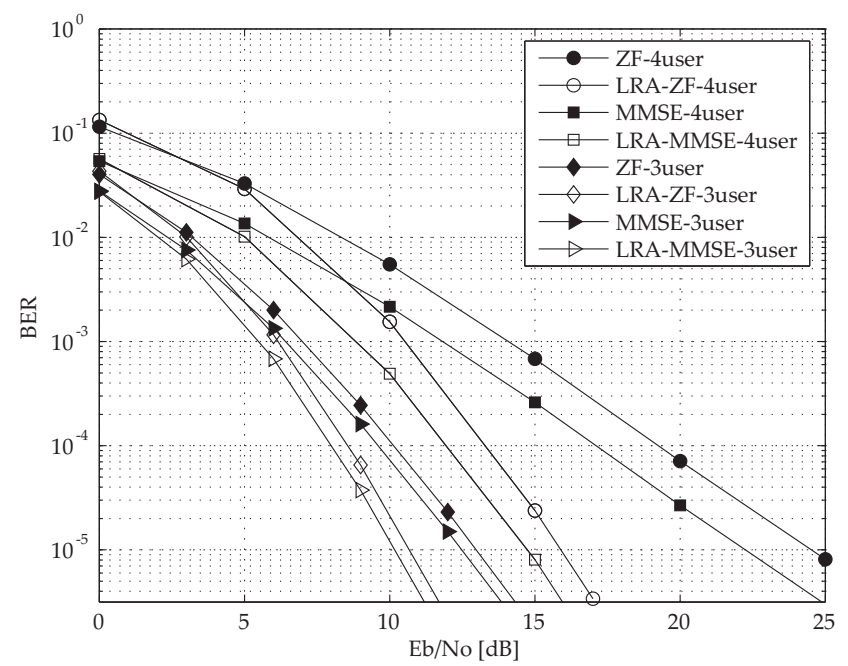

Figure 5. BER performance comparison of LRA ZF and MMSE detection.

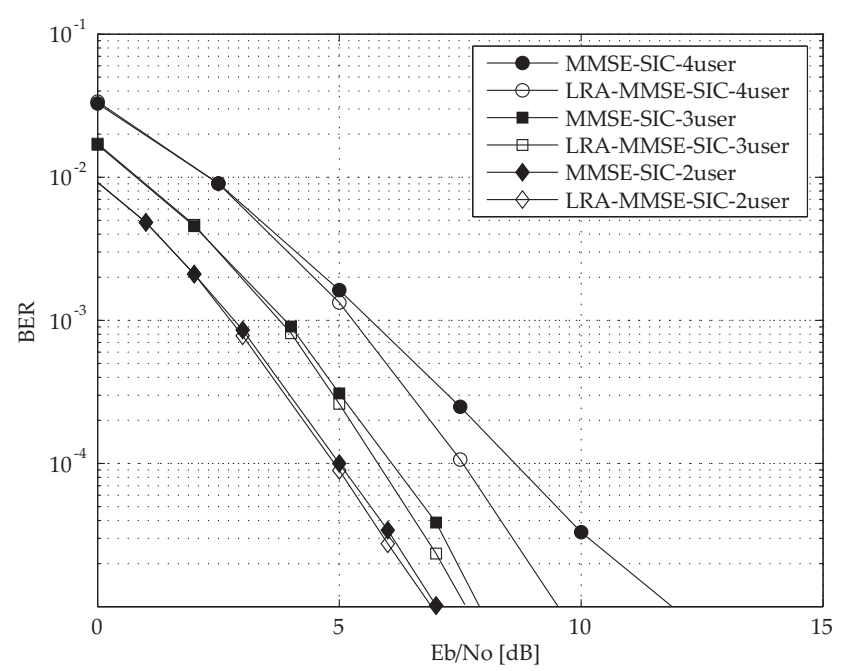

Figure 6. BER performance of LRA-SIC-MMSE detection.

\section{REFERENCES}

[1] S. Verdu, Multiuser Detection, 2nd ed. Cambridge University Press, 1998.

[2] H. Yao and G. W. Wornell, "Lattice-reduction-aided detectors for mimo communication systems," in GLOBECOM'02, vol. 1, November 2002, pp. 424-428.

[3] K. L. Clarkson, W. Sweldens, and A. Zheng, "Fast multiple-antenna differential decoding," IEEE Trans. on Communications, vol. 49, pp. 253-261, February 2001.

[4] S. M. Alamouti, "A simple transmit diversity scheme for wireless communications," IEEE Journal on Selected Areas in Communications, vol. 16, no. 8, pp. 1451-1458, October 1998.

[5] X. N. Tran, T. Fujino, and Y. Karasawa, "An mmse detector for multiuser space-time block coded ofdm," IEICE Transactions on Communications., vol. E88-B, no. 1, pp. 141-149, January 2005.

[6] A. T. Le, X. N. Tran, and T. Fujino, "Combined $\mathrm{ml}$ and mmse multiuser detection for stbc-ofdm systems," IEICE Transactions on Fundamentals, vol. E88-A, no. 10, pp. 29152925, October 2005.

[7] T. D. Nguyen, X. N. Tran, and T. Fujino, "Latticereduction-aided multiuser detection for space-time block coded multiple antenna systems," in The 28th Symposium on Information Theory and Its Applications (SITA2005), 
Okinawa, Japan, November 2005.

[8] J. Eilert and D. Liu, "Lattice-reduction aided multi-user stbc decoding with resource constraints," in The IEEE 18th International Symposium on Personal, Indoor and Mobile Radio Communications (PIMRC2007), September 2007, pp. 1-5.

[9] C. H. An, T. Lee, J. Yang, and D. K. Kim, "Low complexity lattice reduction scheme for stbc two-user uplink mimo systems," EURASIP Journal on Wireless Communications and Networking, vol. 2011:76, pp. 1-10, 2011.

[10] V. Tarakh, A. Naguib, H. Jafakhani, and A. A. Calderbank, "Combined array processing and space-time coding," IEEE Transactions on Information Theory, vol. 45, no. 4, pp. 1121-1128, May 1999.

[11] E. N. Onggosanusi, A. G. Dabak, and T. M. Schmidl, "High rate space-time block coded scheme: Performance and improvement in correlated fading channels," in Proc. of IEEE Wireless Communications and Networking Conference, vol. 1, March 2002, pp. 194-199.

[12] R. F. H. Fischer and C. Windpassinger, "Real- vs. complex-valued equalisation in v-blast systems," Electronics Letters, vol. 39, no. 5, pp. 470-471, March 2003.

[13] A. K. Lenstra, H. W. Lenstra, and L. Lovász, "Factoring polynomials with rational coefficients," Mathematische Annalen, vol. 261, no. 515-534, 1982.

[14] E. Viterbo and E. Biglieri, "A universal decoding algorithm for lattice codes," in 14th Colloque sur le traitement du signal et des images, FRA, 1993. GRETSI, Groupe d'Etudes du Traitement du Signal et des Images, 1993.

[15] L. Hanzo, B. J. C. M. Münster, and T. Keller, OFDM and MC-CDMA for Broadband Multi-User Communications, WLANs and broadcasting. IEEE Press, John Wiley\&Sons, Ltd., 2003.

[16] B. Hassibi, "An efficient square-root algorithm for blast," in IEEE International Conferenec on Acoustic, Speech, Signal Processing, Istanbul, Turkey, 2000, pp. 5-9.

[17] D. Wübben, V. K. R. Bohnke, and K. D. Kammeyer, "Mmse extention of v-blast based on sorted qr decomposition," in The 58th IEEE Vehicular Technlogy Conference, vol. 1, Fall 2003, pp. 508-512.

[18] C. Windpassinger and R. F. H. Fischer, "Low-complexity near maximum likelihood detection and precoding for mimo systems using lattice reduction," in IEEE Information Theory Workshop, Germany, March-April 2003, pp. 345-348.

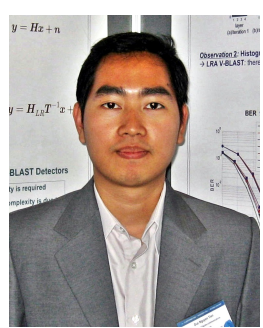

Tien Duc Nguyen received his bachelor of engineering degree in electronics and telecommunication from Hanoi University of Technology, Vietnam, in 2001, master of engineering in Mobile communications from Aalborg University, Denmark in 2004. During the period from 2005 to 2008 he was a doctorate student at The University of Electro-Communications, Japan. Mr. Nguyen passed away in 2009 due to an accident.

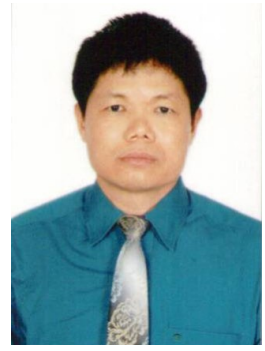

Quang Duc Le was born in Ha Tay, Ha Noi. He obtained his Engineer in Radio-Electronics from VAAZ Academy (Czech Republic) in 1983, Master of Engineering in Telecommunications and Computer Networks from Royal Melbourne Institute of Technology (Australia) in 1998. He is currently working at Institute of Information Technology, The Military Institute of Science and Technology, Vietnam. His research lies in the areas of system integration, MIMO systems, wireless networks and

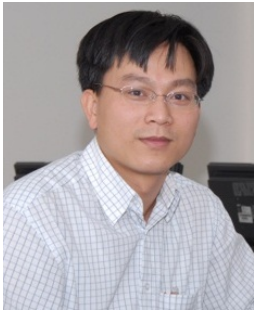

Xuan Nam Tran is currently an associate professor at Department of Communications Engineering at Le Quy Don Technical University Vietnam. He received his master of engineering (ME) in telecommunications engineering from University of Technology Sydney, Australia in 1998, and doctor of engineering in electronic engineering from The University of Electro-Communications, Japan in 2003. From November 2003 to March 2006 he was a research associate at the Information and Communication Systems Group, Department of Information and Communication Engineering, The University of ElectroCommunications, Tokyo, Japan. Dr. Tran research interests are in the areas of adaptive antennas, space-time processing, space-time coding and MIMO systems. He is the author and co-author of more than thirty technical papers published in international journals and conference proceedings. Dr. Tran is a recipient of the 2003 IEEE AP$\mathrm{S}$ Japan Chapter Young Engineer Award. He is a member of IEEE, IEICE, and the Radio-Electronics Association of Vietnam.

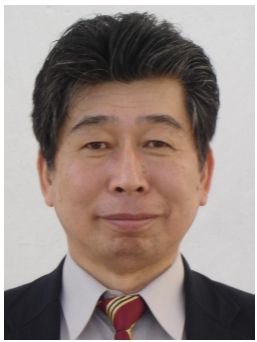

Tadashi Fujino was born in Osaka, Japan on 15 July, 1945. He received B.E. and M.E. degrees in electrical engineering and Dr. Eng. degree in communication engineering from Osaka University, Osaka, Japan, in 1968, 1970, and 1985, respectively.

Since April 2011, he has been Professor Emeritus of The University of ElectroCommunications (UEC), Tokyo, Japan. From April 2000 to March 2011, he was an ordinary professor in wireless communications at Department of Information and Communication Engineering and Informatics, Graduate School of Informatics and Engineering, The UEC, Tokyo, Japan. Before then, he had been with Mitsubishi Electric Corporation, Tokyo, Japan since 1970, where he devoted in R\&D in the wireless communications area such as digital satellite communications and digital land mobile communications. His major works include the feasibility study and the hardware development of the $120 \mathrm{Mbps}$ trellis coded (TC) 8-PSK burst modem for TDMA system. This work was done at INTELSAT's request, and is the first development in the world. In the above feasibility study and the hardware development, he made two innovative inventions: one is the "pipe-lined Viterbi decoder", which makes possible for TC modems to operate in very high bit rates of hundreds Mbps, and the other is the clock aided carrier recovery system for the coherent detection of M-PSK modulated signal, which generates very little pattern noise. After designing and manufacturing the hardware of the TC burst modem using these inventions, he made field test, which was successfully done. By his two inventions and the field test results, it is verified that the high bit rate TC burst modem should be feasible to design. His current interests include the signal detection in MIMO systems such as lattice-reduction aided detection. He wrote a single authored book "Digital mobile communication", and three co-authored books. He received Meritorious Award from the ARIB (The Associate of Radio Industries and Businesses of Japan) of MPT of Japan, in 1997. Dr. Fujino is a Fellow of IEEE and a member of IEICE. 